\title{
Memórias de familiares de profissionais da saúde mortos pela COVID-19 no Brasil
}

\author{
Memories of Family members of health professionals killed by COVID-19 in Brazil \\ Recuerdos de familiares de profesionales de la salud asesinados por COVID-19 em Brasil
}

Recebido: 03/02/2021 | Revisado: 12/02/2021 | Aceito: 15/02/2021 | Publicado: 23/02/2021

\author{
Ruth Noêmia Paula Biork \\ ORCID: https://orcid.org/0000-0003-0760-7473 \\ Universidade do Estado de Mato Grosso, Brasil \\ E-mail: ruth.biork@unemat.br \\ Vagner Ferreira do Nascimento \\ ORCID: https://orcid.org/0000-0002-3355-163X \\ Universidade do Estado de Mato Grosso, Brasil \\ E-mail: vagnernascimento@unemat.br
}

\begin{abstract}
Resumo
Objetivou conhecer as memórias de familiares de profissionais da saúde mortos pela COVID-19 no Brasil. Trata-se de estudo exploratório e qualitativo. Os dados foram coletados em julho de 2020, na página digital Inumeráveis, memorial dedicado à história de cada uma das vítimas do coronavírus no Brasil. Para organização e análise dos dados textuais, utilizou-se o software IRAMUTEQ versão 0.7 Alpha 2 e R Versão 3.2.3, com análise de Classificação Hierárquica Descendente. Entre os depoimentos, a maioria correspondia a profissionais do sexo masculino, com faixa etária entre $22 \mathrm{e}$ 92 anos. Houve uma prevalência de depoimentos da região Sudeste do Brasil. As memórias dos profissionais mortos pela COVID-19 no Brasil foram distribuídas em duas grandes dimensões. A primeira se refere à aspectos profissionais (construção e atuação), e a segunda associada a memórias sobre o valor humano, marcas pessoais, hábitos e papéis desempenhados pelos profissionais no contexto familiar. Através das memórias, o imaginário positivo nutre a perseverança dos amigos e familiares diante da morte de seus entes queridos, e fornece elementos que podem ser inclusos em programas de acolhimento e suporte terapêutico, auxiliando os profissionais da área na escolha de melhores intervenções.
\end{abstract}

Palavras-chave: Núcleo familiar; Pessoal da saúde; Infecções por Coronavírus; Morte; Emoções.

\begin{abstract}
It aimed to know the memories of family members of health professional killed by COVID-19 in Brazil. Incluir o resumo em inglês. This is an exploratory and qualitative study. The data were collected in July 2020, on the innumerable digital page, a memorial dedicated to the history of each of the victims of the coronavirus in Brazil. For organization and analysis of textual data, the software IRAMUTEQ version 0.7 Alpha 2 and R Version 3.2.3 was used, with analysis of Descending Hierarchical Classification. Among the testimonies, most corresponded to male professionals, aged between 22 and 92 years. There was a prevalence of testimonials from the Southeast region of Brazil. The memories of the professionals killed by COVID-19 in Brazil were distributed in two major dimensions. The first refers to professional aspects (construction and performance), and the second associated with memories about human value, personal marks, habits and roles played by professionals in the family context. Through memories, the positive imagery nourishes the perseverance of friends and family in the face of the death of their loved ones, and provides elements that can be included in welcoming and therapeutic support programs, helping professionals in the area in choosing the best interventions.
\end{abstract}

Keywords: Nuclear family; Health personnel; Coronavirus infections; Death; Emotions.

\section{Resumen}

Tenía como objetivo conocer los recuerdos de familiares de profesionales de la salud asesinados por COVID-19 en Brasil. Este es un estudio exploratorio y cualitativo. Los datos fueron recolectados en julio de 2020, en la innumerable página digital, un memorial dedicado a la historia de cada una de las víctimas del coronavirus en Brasil. Para la organización y análisis de los datos textuales se utilizó el software IRAMUTEQ versión 0.7 Alpha 2 y R Versión 3.2.3, con análisis de Clasificación Jerárquica Descendente. Entre los testimonios, la mayoría correspondió a profesionales del sexo masculino, con edades comprendidas entre los 22 y los 92 años. Hubo un predominio de testimonios de la región sureste de Brasil. Los recuerdos de los profesionales asesinados por COVID-19 en Brasil se distribuyeron en dos grandes dimensiones. El primero se refiere a aspectos profesionales (construcción y desempeño), y el segundo asociado a memorias sobre el valor humano, las marcas personales, los hábitos y los roles que desempeñan los profesionales en el contexto familiar. A través de los recuerdos, el imaginario positivo nutre la perseverancia de amigos y familiares ante la muerte de sus seres queridos, y aporta elementos que pueden ser incluidos en los programas de acogida y apoyo terapéutico, ayudando a los profesionales del área a elegir las mejores intervenciones.

Palabras clave: Núcleo familiar; Personal de salud; Infecciones por Coronavirus; Muerte; Emociones. 


\section{Introdução}

No final do ano de 2019, em Wuhan, na República Popular da China, foram relatados os primeiros casos de COVID19, doença causada por um novo tipo de coronavírus, o SARS-CoV-2. Em 11 de março de 2020, devido à alta propagação do vírus, a Organização Mundial de Saúde (OMS), caracterizou a COVID-19 como uma pandemia, já que haviam surtos da doença em inúmeros países e regiões do mundo (Organização Pan-Americana de Saúde [OPAS], 2020). No Brasil, o primeiro caso de COVID-19 foi confirmado em 26 de fevereiro de 2020, desde então, o país vem enfrentando aumento no número de contaminados e de mortos, chegando a 226.309 óbitos até o dia 02 de fevereiro de 2021 (Ministério da Saúde, 2021).

Os profissionais da saúde também integram as estatísticas de infectados e óbitos pela COVID-19 (Campos \& Leitão, 2021; Díaz-Pérez, 2020). Na linha de frente e respeitando as medidas protetivas, distanciam da interação face a face tanto entre os pacientes como entre os membros da sua rede socioafetiva, interações de extrema importância para o cuidado humanizado, sustentação da sociabilidade, arrimo e segurança emocional. Quando estes profissionais se tornam vítimas da COVID-19, além da interrupção dos chamados "rituais de despedida" dos pares, família e amigos (Ingravallo, 2020), o contato que estava temporariamente distante, perpetua-se.

Invisíveis e constantemente violentados no cotidiano do trabalho, os profissionais da saúde passaram a heróis nessa pandemia, recebendo aplausos e reconhecimentos nos mais diversos países (Passos \& Prazeres, 2020). E em consequência, diante dos óbitos desses profissionais a consternação popular foi ampliada, pelo reconhecimento do altruísmo e valores que possuem em meio a um cenário de caos e condições precárias de trabalho (Nascimento et al., 2020a).

Mesmo que não seja homogênea, a elaboração do luto pelos familiares dos profissionais da saúde tende a ser um processo longo e complexo, pois além da combinação da comoção social, subtração e desligamento permanente do contato presencial, não há chances de serem confortados e oferecerem apoio às pessoas próximas. Ainda que alguns consigam elaborar o seu luto e estabelecerem rearranjos, outros vivenciarão o luto complicado, permeado por pensamentos perturbadores, prejuízos nas relações interpessoais e até mesmo percepção de vida sem sentido (Ingravallo, 2020; Wallace et al., 2020).

Com essa experiência, há familiares que buscam recursos terapêuticos que oportunizem reforço positivo a travessia desse momento de perda, sofrimento e medo da COVID-19. Uma dessas estratégias que vem sendo proposta e utilizada é a criação de memórias on line, permitindo que familiares, amigos e sociedade possuam um espaço para condolências, homenagens e reencontro com recordações. Esse compartilhamento de momentos e lembranças, entendido como uma estratégia para enfrentamento do luto durante a pandemia, favorecem as manifestações de amparo, abrandamento e conexão emocional (Fundação Osvaldo Cruz [FIOCRUZ], 2020; Meyer, 2016; Social Science In Humanitarian Action, 2020).

Ao compreender as repercussões danosas em relação aos óbitos e particularmente daqueles que perderam suas vidas em prol do cuidado humano durante a pandemia, questiona-se quais foram as memórias deixadas por esses profissionais? Assim, o estudo teve como objetivo conhecer as memórias de familiares de profissionais da saúde mortos pela COVID-19 no Brasil.

\section{Metodologia}

Trata-se de estudo exploratório e qualitativo, baseado em dados públicos e gratuitos, seguindo o protocolo internacional Consolidated Criteria For Reporting Qualitative Research (COREQ). Os dados foram coletados em julho de 2020, diretamente na página digital Inumeráveis, memorial eletrônico dedicado à história de cada uma das vítimas do coronavírus no Brasil, criada pelo artista Edson Pavoni e o empreendedor social Rodrigo Oliveira. Através deste memorial, familiares e/ou amigos respondem a um questionário sobre a vítima. Esse questionário é automaticamente direcionado para uma rede de jornalistas, todos voluntários. Com base nas informações fornecidas, um dos jornalistas estrutura um texto tributo para cada vítima, que será então inserido nesse memorial. $\mathrm{O}$ acesso ao memorial é aberto, disponibilizado através do sítio eletrônico https://inumeraveis.com.br/. 
Os dados utilizados se referem aos depoimentos inseridos nesse ambiente virtual, a partir de 30 de abril de 2020, data de início da atividade dessa plataforma digital. Foram incluídos no estudo, os depoimentos alusivos aos profissionais da saúde atuantes no território brasileiro. E, excluiu-se os depoimentos que se referiam as profissionais gestantes, pois vivenciam a COVID-19, de modo diferente daqueles que estão na linha de frente. A amostragem do estudo foi do tipo censitária, incorporando todos os registros referentes aos profissionais da saúde, o que totalizou 126 depoimentos.

Para organização e análise dos dados textuais, utilizou-se o software IRAMUTEQ versão 0.7 Alpha 2 e R Versão 3.2.3, com análise de Classificação Hierárquica Descendente (CHD), seguindo três etapas: preparação e codificação do texto inicial, classificação e interpretação das classes (Kami et al., 2016). Os resultados foram apresentados em dendograma e gráfico de análise fatorial de correspondência (AFC).

O dendograma possibilita visualizar quais palavras obtiveram maior porcentagem quanto à frequência média entre si e diferente entre elas. Já, o gráfico AFC permite identificar uma estrutura latente em uma totalidade de relações, na qual múltiplas variáveis atuam ao mesmo tempo. Cada variável recebe um valor específico, e a proximidade entre elas resulta na criação de conjuntos, que correspondem os dois eixos do plano fatorial (Kami et al., 2016).

Este estudo seguiu todos aspectos éticos em pesquisa que utiliza dados abertos e públicos, respeitando todas as diretrizes da Resolução 466/2012 e 510/2016 do Conselho Nacional de Saúde (CNS).

\section{Resultados}

O preparo do texto consistiu na transcrição dos depoimentos, formando o corpus de análise. Foram utilizados 126 depoimentos, que quando organizados geraram 126 Unidade de Contexto Inicial (UCI). Cada uma foi separada através de linha de comando, compreendendo apenas uma variável (D), escolhida conforme o número dado a cada depoimento (*****D_1, **** *D_2 até *****D_126). Após a transcrição, realizada através do LibreOffice Writer, do pacote LibreOffice.org, o arquivo foi salvo como documento de texto usando a codificação de caracteres no padrão UTF-8 (Unicode Transformation Format 8 bit codeunits).

Entre os depoimentos, $57,1 \%$ (72) correspondiam ao sexo masculino, com faixa etária entre 22 e 92 anos. Houve uma prevalência de depoimentos na região sudeste do Brasil, acumulando 53,1\% (67) do total de depoimentos. No dendograma, o corpus foi dividido em dois subcorpus, sendo a classe 1 com 27 Unidades de Contexto Elementar (UCE), classe 2 com 17 UCE, classe 3 com 31 UCE, classe 4 com 22 UCE e classe 5 com 29 UCE. Entre elas, a classe 3 possui a maior representação social $(24,5 \%)$ do corpus, em seguida a classe $5(23,2 \%)$ e classe $1(21,3 \%)$ (Figura 1$)$. 
Figura 1 - Dendograma com a porcentagem de UCE em cada classe e palavras com maior qui-quadrado.

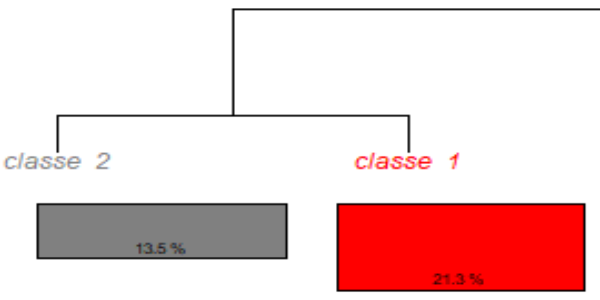

frente

linha

combate

pandemia

trabalhador

risco

grupo

servidor

ignez

mesmo

aposentadoria

maio

entrada

social

contrair

cuidado

doente

funcionária

trabalhar
janeiro
realizar
hospital
ano
sonho
rio
medicina
formar
estudar
enfermagem
público
exercer
área
faculdade
saúde

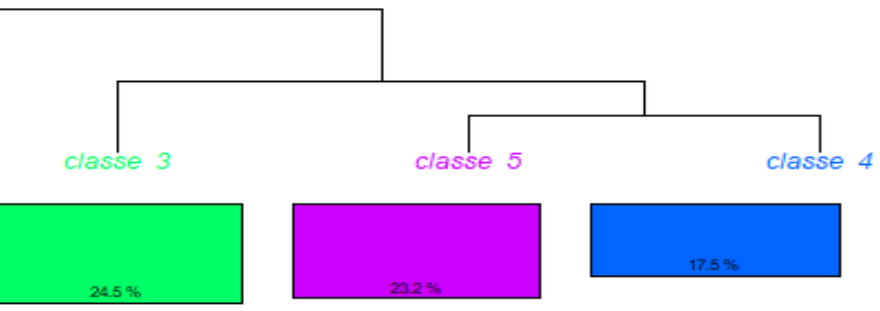

próximo

viver

adorar

gostar

depoimento

amor

esposo

fé

cheio

maneira

puro

frase

dançar

garra

momento

generosidade quando

tudo

pedir

vez

dor

café

porque

criança

falar

perto

comer

tomar

simples

ana

apaixonado

filho

\section{terra}

saudade

aqui

partir

deixar

legado

vazio

mensagem

olhar

amigo

enviar

voz

sentimento

anjo

ficar

eterno

Fonte: Autores.

Na classe 1, pode-se perceber a relação com o processo de formação e perspectiva de atuação dos profissionais da saúde. A classe 2, volta-se aos riscos destes trabalhadores na linha de frente no combate à COVID-19. A classe 3 demonstra as características e adjetivos desses profissionais. Na classe 4 há referência ao que restou/ficou após a partida do profissional. E a classe 5 , reforça as memórias da infância à dor da perda.

No plano fatorial há quatro quadrantes e cinco comunidades, que apesar de terem padrões semânticos diferentes, possuem similaridade de modo que alguns grupos de palavras tenham aproximação e inter-relação em diferentes áreas do plano (Figura 2). 
Figura 2 - Representação Fatorial das classes.

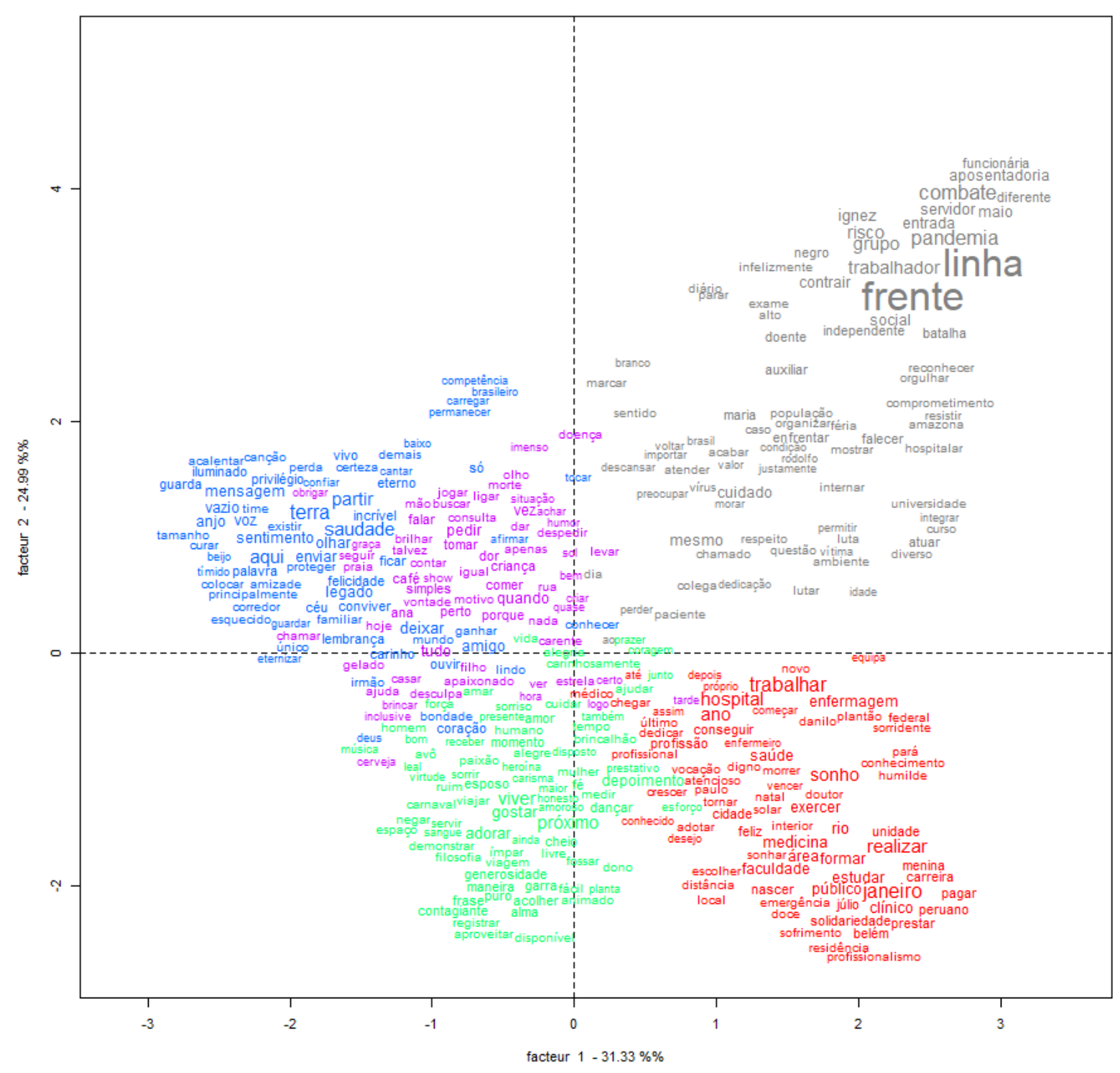

Fonte: Autores.

No quadrante I, apresenta-se a classe 2, a mais isolada no plano cartesiano. Embora a classe 1 (quadrante IV), esteja nesse mesmo lado do plano e consequentemente possua correspondência lexical, remetem a campos semânticos distintos, logo sem aproximação entre elas. No quadrante II, as classes 4 e 5 exibem movimento de união, e ligação/complementariedade com a classe 3 (quadrante III). As classes do eixo x corresponderam a maior variância das UCE $(31,33 \%)$.

\section{Discussão}

As memórias dos profissionais da saúde mortos pela COVID-19 no Brasil foram distribuídas em duas grandes dimensões. A primeira (classe 1 e 2) se refere à aspectos profissionais (construção e atuação), e a segunda (classe 3, 4 e 5) associada a memórias sobre o valor humano, marcas pessoais, hábitos e papéis desempenhados pelos profissionais no contexto familiar.

Em relação a primeira dimensão, essas memórias ao relacionarem a trajetória e representatividade de uma profissão que transformou a vida do seu familiar, reforça o que alguns autores afirmam, que para além de aspectos esperados em uma profissão, 
como a inserção no mercado de trabalho, independência e estabilidade financeira, esse caminho de estudos e trabalho propicia o compartilhamento de sonhos, e sobretudo o vislumbrar de espaços conquistados e satisfação no exercício profissional (Buscacio \& Soares, 2017; Nogueira et al., 2020; Sigaud et al., 2016). Essa inspiração e realização mútua (família e familiar), durante a pandemia da COVID-19 deixou de ser um sonho e assumiu o sentimento de saudade e admiração.

Por outro lado, esse reforço positivo em relação a construção do profissional para a família, baseia-se na ausência de reconhecimento real do trabalhador do setor saúde, que ficou mais evidente com a chegada da COVID-19 (Lopes, 2020). Em consequência, as menções à profissão como um sonho alcançado, revelam o valor de tornar-se um cuidador, sujeitos plurais tanto profissionalmente quanto em suas histórias de vidas, mas que se reduzem ao grupo de anonimatos quando entram para a estatística de óbitos (Medina, 2020).

Os familiares também remetem suas lembranças à atmosfera de riscos, vivenciada com intensidade pelos profissionais, que a todo momento mesmo sob atenção redobrada, estão vulneráveis a não retornar para suas casas, em consequência também não reencontrarem com a família (Shigemura et al., 2020). Essa relação embora seja revestida de compreensão e afeto, reverbera no seio familiar a fadiga física e mental que os profissionais experimentam em suas rotinas, e no momento em que a família poderia ser o esteio se vê forçada a lidar com a impossibilidade de oferecer apoio, em razão da testagem positiva do profissional (Cardoso, Silva et al., 2020; Kang et al., 2020).

Além disso, a família percebe duas grandes ameaças, a possível perda do familiar e a perda do senso de controle sobre os acontecimentos, pois a imprevisibilidade da infecção não acompanha a mesma velocidade de se preparem para possíveis consequências danosas (Cardoso, Silva et al., 2020). E isso ocorre, pois, o profissional da saúde pode servir como um funil através do qual sua rotina e riscos vividos diariamente frente à COVID-19 se infiltram no funcionamento e bem-estar da família (Prime et al., 2020).

Estudo realizado com enfermeiros em Portugal (Cardoso, Martins et al., 2020), indica que os profissionais antes da pandemia já possuíam ansiedade e medo desencadeados pela própria morte, mas com a pandemia isso agravou, realidade semelhante no Brasil (Nascimento et al., 2020a). No entanto, mesmo esses enfermeiros tendo a atitude de evitar falar sobre a morte (Cardoso, Martins et al., 2020), seus pares, principalmente os familiares, não ficam isentos desse sofrimento.

Nesse contexto, além de estar envolvida sinergicamente com seu familiar, a família torna-se plateia que assiste angustiada essa rotina de trabalho, pois tem a convicção que estar na linha de frente é viver o risco do desentrincheiramento, sem suprimentos de recursos, por exemplo. Esses motivos podem ficar latentes nas memórias dos familiares (Huang et al., 2020), com a sensação de impotência pela suscetibilidade do trabalhador (Teixeira et al., 2020) e pelo descaso dos gestores e Governos (Ferreira et al., 2020).

Em contraponto, as menções de fé, amor e generosidade realçam elementos que auxiliam na resiliência desses familiares, na medida que ao possuírem lembranças positivas há compreensão do valor do ser humano, que pode contribuir para um sentimento de percepção de sua própria condição atual (Zanon et al., 2020). Esse processo de adjetivação de memórias traduz as mudanças ocorridas nas relações familiares em razão da pandemia, com apelo ao fortalecimento das estruturas familiares, bem como o enaltecimento do espírito fraterno e solidário entre os pares (Schmidt et al., 2020).

Nesse âmbito, a associação da fé como lembrança do profissional da saúde pode inspirar e ajudar o familiar a viver essa perda, pois pensar na fé tende a gerar um sentimento de esperança, que determina outras formas de enfrentamento. Essas novas formas podem substituir a sensação de fatalismo e medo sobre o que pode ocorrer, na vivência do ninho vazio e/ou na percepção de solidão sem o ente querido (Modell \& Kardia, 2020).

Embora o distanciamento social venha ampliando os sofrimentos das famílias durante a pandemia, o contato físico e emocional concedido dentro de uma mesma casa, cria elos poderosos de amor e partilha. Logo, diante da morte, ainda que haja reações diversas à essa experiência, pela personalidade, estilo de enfrentamento, relacionamento com o falecido e circunstâncias 
da morte, a família pode se abastecer na união e viver próximo de como era seu familiar (Lebow, 2020).

Nos depoimentos, os familiares também mencionaram alguns legados deixados pelos entes queridos, desde o olhar à voz. Esses legados não se restringem a dimensão exclusivamente afetiva que o familiar representava, mas ao significado dos seus papéis junto a família (Johnson \& Johnson, 2020).

A morte prematura (Silva et al., 2020) e interrupção abrupta da realização dos papéis sociais que eram desenvolvidos pelo falecido, traz para a família a necessidade de reorganização e de aprendizagem de tais papéis. Um estudo realizado em São Paulo (BR), aponta que durante o processo do luto, há a necessidade de revezar os papéis sociais entre os integrantes de uma determinada família, afim de manter as atividades que estruturavam o cotidiano da família enlutada (Aciole \& Bergamo, 2019).

O importante papel social exercido pelos profissionais da saúde durante a pandemia, chamados de heróis, para as famílias enlutadas representaram a materialização de anjos na terra, que partiram deixando um vazio. Pesquisa conduzida com mães goianas aponta que esse vazio versa sobre o que chamam de presença-ausente, ausente por não poder tocar no familiar e presente pelas lembranças (Assis, Motta \& Soares, 2019), que no presente estudo se manifesta com a saudade e o imaginário de suas marcas pessoais.

No momento do luto, inicia-se um processo de idealização dos modos de vida do ente falecido, esse processo de idealização serve como estratégia de reparação, visando mostrar que não apenas houve a perda de alguém, mas que essa perda não pode ser negada. Nesse sentido, a idealização é uma ferramenta que mantém viva na memória dos familiares os hábitos do falecido (Pereira \& Pires, 2018).

Conforme os depoimentos, os pequenos detalhes no jeito de ser e viver, como a forma de falar, a bebida preferida, o lugar que o ente querido mais frequentava, tornam-se agora parte do conjunto que representa a dimensão emocional dos vínculos familiares. Estudo sobre obituário, mostra que a retórica fúnebre formada por essas lembranças do gostar e ser do falecido, visa homenagear e dar à família, amigos e entusiastas o seu novo lugar social, reforçando a dimensão identitária com uma mensagem de afeto e reconhecimento para o presente e futuro (Silva, 2019).Esses profissionais da saúde que perderam suas vidas no combate à COVID-19, deixam entre seus colegas de trabalho a coragem em não abandonar à luta em prol da humanidade (Nascimento et al., 2020b) e o espírito de vanguarda no cuidado do ser humano, e para a família e amigos a certeza que serão eternamente lembrados, em uma nova experiência existencial, a vivência interna da pandemia e seus rompimentos.

Diante disso, as lembranças dos familiares do presente estudo, funcionam como uma estratégia para a preservação da presença do familiar e permite que a história da pessoa seja contada da maneira como preferem se lembrar (Fareez, 2019), tornando um recurso terapêutico com possibilidade de sustentar sentimentos (Gonçalves \& Bittar, 2016), e revisitar reminiscências que acolham a dor e gerem fortaleza.

Como limitações desse estudo, destacam-se as características dos dados utilizados, que não permite identificar qual a categoria específica do profissional da saúde, assim como a possibilidade dos familiares ou amigos não terem conseguido expressar pelos depoimentos todo o significado da perda e representatividade do ente querido. Para tanto, dar visibilidade a essas memórias, permite repensar a valorização atribuída aos profissionais no contexto da pandemia pelos familiares, ser alento frente aos sofrimentos e ao mesmo tempo, reforçar a precariedade das condições de trabalho e vulnerabilidade desses profissionais no Brasil.

\section{Considerações Finais}

As principais memórias dos familiares de profissionais da saúde mortos pela COVID-19 no Brasil se relacionaram a trajetória de formação e aos riscos diários da atuação do profissional durante a pandemia, e as características da história de vida e participação no seio familiar. Esses apontamentos sinalizam caminhos para apoiar e auxiliar na ressignificação e numa melhor elaboração do luto, assim como constituir novas e significativas formas de interação social e dinâmica da família, baseada na 
interação de lembranças positivas do familiar falecido. Pode fornecer também, elementos para serem inclusos em programas de acolhimento e suporte terapêutico, direcionando os profissionais da área de saúde mental na escolha de melhores intervenções para assistir essas famílias.

Ainda não há estudos que indiquem ou mensurem o impacto da pandemia da COVID-19 no bem-estar de famílias enlutadas, especificamente na perda de um familiar profissional da saúde. Assim, o presente estudo é pioneiro na identificação de aspectos que revelam, através de memórias, o imaginário que nutre a vivência de familiares diante da morte de seus entes queridos, sendo fundamental novas pesquisas para aprofundar as perspectivas e impactos do processo de morte e morrer no contexto familiar durante e pós pandemia.

\section{Referências}

Aciole, G. G., \& Bergamo, D. C. (2019). Cuidado à família enlutada: uma ação pública necessária. Revista Saúde em Debate, $43(122)$, 805-818.

Assis, G. A. P., Motta, H. L., \& Soares, R. V. (2019). Falando sobre presenças-ausentes: vivências de sofrimento no luto materno. Revista do NUFEN, 11(1), 39-54.

Buscacio, R. C. Z., \& Soares, A. B. (2017). Expectativas sobre o desenvolvimento da carreira em estudantes universitários. Revista Brasileira de Orientação Profissional, 18(1), 69-79.

Campos, A. C. V., \& Leitão L. P. C. (2021). Letalidade da COVID-19 entre profissionais de saúde no Pará, Brasil. Journal Health NPEPS, 6(1), 22-34.

Cardoso, E. A. O., Silva, B. C. A., Santos, J. H., Lotério, L. S., Accoroni, A. G., \& Santos, M. A. (2020). Efeitos da supressão de rituais fúnebres durante a pandemia de COVID-19 em familiares enlutados. Revista Latino Americana de Enfermagem, 28 , e3361.

Cardoso, M. F. P. T., Martins, M. M. F. P. S., Ribeiro, O. M. P. L., Pereira, V. L. S. C., Pires, R. M. F., \& Santos, M. R. (2020). Atitudes dos enfermeiros gestores face à morte: repercussões da pandemia por COVID-19. Journal Health NPEPS, 5(2), $42-59$.

Díaz-Pérez, G. (2020). La pandemia de COVID-19 y sus violencias en América Latina. Journal Health NPEPS, $5(2), 1-7$.

Fareez, M. (2019). A 'certidão de vida': uma ferramenta para trabalhar o luto em Singapura. Nova Perspectiva Sistêmica, 28(63), 5-20.

Ferreira, S. R. S., Mai, S., Périco, L. A. D., \& Micheletti, V. C. D. (2020). O processo de trabalho da enfermeira, na atenção primária, frente à pandemia da COVID-19. In S. S. S. Teodósio, \& S. S. Leandro (Orgs.), Enfermagem na Atenção Básica no Contexto da Covid-19 (pp. 19-26). ABen/DEAB.

Fundação Oswaldo Cruz. (2020). Saúde mental e atenção psicossocial na pandemia COVID-19: processo de luto no contexto da COVID-19. https://www.fiocruzbrasilia.fiocruz.br/wp-content/uploads/2020/04/Sa\%c3\%bade-Mental-e-Aten\%c3\%a7\%c3\%a3o-Psicossocial-na-Pandemia-Covid-19processo-de-luto-no-contexto-da-Covid-19.pdf.

Gonçalves, P. C., \& Bittar, C. M. L. (2016). Estratégias de enfrentamento no luto. Mudanças - Psicologia da Saúde, 24(1), 39-44.

Huang, L., Lin, G., Tang, L., Yu, L., \& Zhou, Z. (2020). Special attention to nurses' protection during the COVID-19 epidemic. Critical Care, $24(1)$, 120.

Ingravallo, F. (2020). Death in the era of the COVID-19 pandemic. The Lancet Public Health, 5(5), e258.

Johnson, L. K., \& Johnson, K. (2020). A morte e o luto em tempos de pandemia. Revista Culturas e Fronteiras, 2(2), 1-9.

Kami M. T. M., Larocca L.M., Chaves M. M. N., Lowen I. M. V., Souza V.M.P., \& Goto D. Y. N. (2016). Working in the street clinic: use of IRAMUTEQ software on the support of qualitative research. Escola Anna Nery, 20(3), e20160069.

Kang, L., Li, Y., Hu, S., Chen, M., Yang, C., Yang, B. X., Wang, Y., Hu, J., Lai, J., Ma, X., Chen, J., Guan, L., Wang, G., Ma, H., \& Liu, Z. (2020). The mental health of medical workers in Wuhan, China dealing with the 2019 novel coronavirus. The Lancet Psychiatry, 7(3), e14.

Lebow, J. L. (2020). Family in the age of COVID-19. Family Process, 59(2), 309-312.

Lopes, E. A. B. (2020). Vivências de sofrimento e adoecimento em ambiente de trabalho: uma análise do cotidiano profissional de enfermeiras e enfermeiros num contexto pandêmico em dois centros de referência no atendimento a pacientes de Covid-19. Cadernos de Psicologia Social do Trabalho, 23(2), 218-235.

Medina, C. (2020). Apontamentos do espanto na crise COVID-19. Revista Longeviver, 2(7), 4-8.

Meyer, E. P. (2016). Death in the age of eternity: how Facebook users cope with personal loss. (Masters dissertation, Iwoa State University). Graduate Theses and Dissertations. https://lib.dr.iastate.edu/etd/15772

Ministério da Saúde do Brasil. (2021). Painel Coronavírus. https://covid.saude.gov.br.

Modell, S. M., \& Kardia, S. L. R. (2020). Religion as a health promoter during the 2019/2020 COVID outbreak: view from Detroit. Journal of Religion and Health, 59(5), 2243-2255. 
Research, Society and Development, v. 10, n. 2, e45610212647, 2021

(CC BY 4.0) | ISSN 2525-3409 | DOI: http://dx.doi.org/10.33448/rsd-v10i2.12647

Nascimento, V. F., Hattori, T. Y., \& Terças-Trettel, A.C.P. (2020a). Necessidades pessoais de Enfermeiros durante a pandemia da COVID-19 em Mato Grosso. Enfermagem em Foco, 11(1), 141-145.

Nascimento, V. F., Espinosa, M. M., Neri, M. C., Pinheiro, N., \& Terças-Trettel, A. C. P. (2020b). Impacto da COVID-19 sob o trabalho da enfermagem brasileira: aspectos epidemiológicos. Enfermagem em Foco, 11(1), 24-31.

Nogueira, C. C. C., Soares, A. B., Monteiro, M. C., \& Medeiros, H. C. P. (2020). Habilidades sociais e expectativas acadêmicas em estudantes de enfermagem. Estudos e Pesquisas em Psicologia, 20(1), 99-118.

Organização Pan-Americana de Saúde. (2020). Folha informativa - COVID 19 (doença causada pelo novo coronavírus). https://www.paho.org/bra/index.p hp?o ption=com_content\&view=article\&id=6101:covid $19 \&$ Itemid=875.

Passos, L., \& Prazeres, F. (2020). A Mão que Agrediu Agora Aplaude: A Imagem dos Profissionais de Saúde Frente à Pandemia COVID-19. Gazeta Médica https://www.gazetamedica.pt/COVID-19/2020/03/26/a-mao-que-agrediu-agora-aplaude-a-imagem-dos-profissionais-de-saude-frente-a-pandemia-covid-19/ Acesso em: 21 jul. 2020.

Pereira, S. M., \& Pires, E. F. (2018). As experiências de perdas e luto na contemporaneidade: um estudo bibliográfico. Revista Educação, 13 (1), $200-217$.

Prime, H., Wade, M., \& Browne, D. T. (2020). Risk and resilience in Family well-being during the COVID-19 pandemic. American Psychologist, 75(5), 631643.

Schmidt, B., Crepaldi, M. A., Bolze, S. D. A., Neiva-Silva, L., \& Demenech, L. M. (2020). Saúde mental e intervenções psicológicas diante da pandemia do novo coronavírus (COVID-19). Estudos de Psicologia, 37, e200063.

Shigemura, J., Ursano, R. J., Morganstein, J. C., Kurosawa, M., \& Benedek, D. M. (2020). Public responses to the novel 2019 coronavirus (2019-nCoV) in Japan: Mental health consequences and target populations. Psychiatry and Clinical Neurosciences, 74(4), 281-282.

Sigaud, C. H. S., Souza, N. B., Nobrega, A. A., Toriyama, A. T. M., \& Costa, P. (2020). Motivos de estudantes de enfermagem para a escolha da carreira. Revista Iberoamericana de Educacíon y Investigación em Enfermería, 6(4), 18-24.

Silva, R. C. L., Silva, C. R. L., Machado, D. A., Peregrino, A. A. F., Marta, C. B., Pestana, L. C., Pessanha, C. M., Vianna, E. C. C., \& Meireles, I. B. (2020) Anos de vida perdidos ajustados por incapacidade entre os profissionais de enfermagem devido a infecção pelo COVID-19 no Brasil. Research, Society and Development, 9(8), e439985896.

Social Science In Humanitarian Action Platform. (2020). Key considerations: dying, bereavemente and mortuary and funerary practices in the contexto of COVID-19. https://opendocs.ids.ac.uk/opendocs/bitstream/handle/20.500.12413/15236/SSHAP\%20COVID-19\%20brief.\%20D eath \%20and\%20funerals.pdf?sequence=1\&isAllowed=y.

Teixeira, C. F. S., SOARES, C. M., Souza, E. A., Lisboa, E. S., Pinto, I. C. M., Andrade, L. R., \& Espiridião, M. A. (2020). A saúde dos profissionais de saúde no enfrentamento da pandemia de COVID-19. Ciência e Saúde Coletiva, 25(9), 3465-3474.

Wallace, C. L., Wladkowski, S. R., Gibson, A., \& White, P. (2020). Grief during the COVID-19 pandemic: considerations for palliative care providers. Journal of Pain and Symptom Management, 60(1), e7076.

Zanon, C., Dellazzana-Zanon, L. L., Wechsler, S. M., Fabretti, R. R., \& Rocha, K. N. (2020). COVID-19: implicações e aplicações da psicologia positiva em tempos de pandemia. Estudos de Psicologia, 37, e200072. 